\title{
Quantum-Dot-Based Telecommunication-Wavelength Quantum Relay
}

\author{
J. Huwer, ${ }^{*}$ R. M. Stevenson, J. Skiba-Szymanska, M. B. Ward, and A. J. Shields \\ Toshiba Research Europe Limited, Cambridge Research Laboratory, 208 Cambridge Science Park, \\ Milton Road, Cambridge CB4 OGZ, United Kingdom \\ M. Felle \\ Toshiba Research Europe Limited, Cambridge Research Laboratory, 208 Cambridge Science Park, \\ Milton Road, Cambridge CB4 OGZ, United Kingdom \\ and Centre for Advanced Photonics and Electronics, University of Cambridge, J. J. Thomson Avenue, \\ Cambridge CB3 OFA, United Kingdom \\ I. Farrer ${ }^{\dagger}$ and D. A. Ritchie \\ Cavendish Laboratory, University of Cambridge, J. J. Thomson Avenue, \\ Cambridge CB3 OHE, United Kingdom \\ R. V. Penty \\ Centre for Advanced Photonics and Electronics, University of Cambridge, \\ J. J. Thomson Avenue, Cambridge CB3 OFA, United Kingdom
}

(Received 20 April 2017; published 16 August 2017)

\begin{abstract}
The development of quantum relays for long-haul and attack-proof quantum communication networks operating with weak coherent laser pulses requires entangled photon sources at telecommunication wavelengths with intrinsic single-photon emission for most practical implementations. Using a semiconductor quantum dot emitting entangled photon pairs in the telecommunication $\mathrm{O}$ band, we demonstrate a quantum relay fulfilling both of these conditions. The system achieves a maximum fidelity of $94.5 \%$ for implementation of a standard four-state protocol with input states generated by a laser. We further investigate robustness against frequency detuning of the narrow-band input and perform process tomography of the teleporter, revealing operation for arbitrary pure input states, with an average gate fidelity of $83.6 \%$. The results highlight the potential of semiconductor light sources for compact and robust quantum-relay technology that is compatible with existing communication infrastructures.
\end{abstract}

DOI: $10.1103 /$ PhysRevApplied.8.024007

\section{INTRODUCTION}

Optical quantum communication is of fundamental importance for a wide range of emerging quantum technologies. Its applications range from securing integrity of classical communication channels by means of quantum key distribution (QKD) [1,2] to the link of distributed quantum-information units, important for the scalability of future quantum processor frameworks [3].

Quantum information for quantum-communication purposes is conveniently encoded in photonic qubits which consist of single photons or, more generally, weak coherent optical pulses [4] that can be transmitted over optical fiber. To minimize losses, these systems ideally operate in the standard telecommunication-wavelength bands, enabling point-to-point communication over distances of up to a few hundred kilometers $[5,6]$. Yet, the principal laws of

\footnotetext{
*jan.huwer@crl.toshiba.co.uk

Tresent address: Department of Electronic and Electrical Engineering, University of Sheffield, Sheffield S1 3JD, United Kingdom.
}

quantum mechanics prohibit the duplication of a single qubit, precluding amplification of quantum signals, as required for long-haul transmission in a global quantumcommunication network. Alternative devices have to be developed, namely, quantum relays [7] and quantum repeaters $[8,9]$, to effectively reduce noise in a quantum channel and boost transmission distances. Both systems rely on the distribution of entanglement across the network, which is then employed to teleport bits of quantum information [10]. Most appealing for QKD applications is that security of the quantum channel is unconditionally guaranteed [11], even if the entanglement resource and the device itself are provided by an untrustworthy third party.

Entangled photon sources based on nonlinear processes have enabled pioneering work for the demonstration of photonic quantum teleportation [12] — even, most recently, by using fully independent sources $[13,14]$. Nevertheless, the number statistics of emitted photon pairs from these sources follow a Poissonian distribution. Multi-photon events typically increase error rates, requiring operation at very low intensities or use of sophisticated security protocols [4], which is not desirable for robust applications. By contrast, 
entangled photon sources based on semiconductor quantum dots (QDs) [15-18] have purely sub-Poissonian statistics and can be directly electrically driven [19], making them promising candidates for the development of practical technology required for cost-effective and efficient quantum networks with untrusted nodes.

The recent first implementation of a quantum-dotdriven quantum relay [20] has proven the feasibility of this approach by achieving state-of-the-art high-fidelity operation. Nevertheless, the experiment made use of a conventional QD emitting at short wavelength, suffering from high losses when being transmitted over a long optical fiber and, therefore, not suitable for a scalable architecture. Great effort has been put into the development of QDs emitting at telecommunication wavelengths [21-24], culminating in independent demonstrations of entangled photon emission [25] and interference with laser photons [26]. Here, we report the implementation of a quantum-relay experiment in the telecommunication $\mathrm{O}$ band (close to $1310 \mathrm{~nm}$ ), suitable for secure quantumcommunication applications and, at the same time, fully compatible with the existing communication infrastructure regarding networks and sources.

\section{EXPERIMENTAL SETUP}

At the core of the experiment is an entangled photon-pair source based on a self-assembled InAs/GaAs QD in a $p-i-n$ structure, grown by molecular beam epitaxy, similar to the sample used in Refs. $[25,26]$ but with an improved ultralow QD density $\left(<0.02 \mu \mathrm{m}^{-2}\right)$. The device is operated at $10 \mathrm{~K}$ and is optically excited with continuous-wave $1064-\mathrm{nm}$ laser light at a bias of $0 \mathrm{~V}$. The excitation power is set for equal intensity of the biexciton $(2 X)$ and exciton $(X)$ spectral lines, measured with an InGaAs array in a grating spectrometer. The carefully chosen operation conditions result in a coherence time of $95 \pm 8$ ps for the $2 X$ photons, measured with a Mach-Zehnder interferometer. This coherence is sufficient to guarantee indistinguishability of the photons within the timing resolution of our photon-detection setup, as required for the Bell-state measurement in the teleporter. Emission from the QD is coupled to a standard single-mode fiber using a confocal-microscope setup $(\mathrm{NA}=0.68)$ and entangled photons from the $2 X$ and $X$ states close to the center of the telecommunication $\mathrm{O}$ band [Fig. 1(a)] are spectrally isolated with a transmission grating.

Photon entanglement generated from the optical cascade of the doubly excited $2 X$ state via emission from intermediate $X$ levels is described by the maximally entangled state

$$
|\Phi(\tau)\rangle=\frac{1}{\sqrt{2}}\left[\left|H_{2 X} H_{X}\right\rangle+\exp \left(\frac{i S \tau}{\hbar}\right)\left|V_{2 X} V_{X}\right\rangle\right],
$$

where $H$ and $V$ denote horizontal and vertical photon polarization. $S$ accounts for the so-called exciton-spin splitting which is present in most QDs and is related to

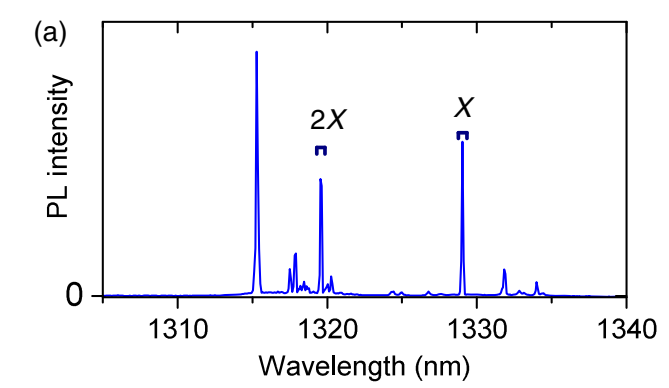

(b)

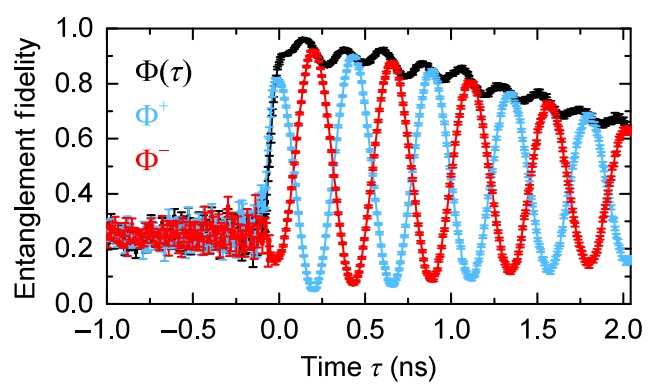

FIG. 1. (a) Photoluminescence spectrum of quantum-dot emission in the telecommunication $\mathrm{O}$ band with indicated recombination lines of biexciton $(2 X)$ and exciton $(X)$ photons. (b) Measured fidelity of photon pairs to the maximally entangled $\Phi^{+}$(blue curve) and $\Phi^{-}$(red curve) Bell states, and a timeevolving state as a function of time delay $\tau$ between $2 X$ - and $X$-photon emission. Error bars indicate the propagated errors from Poissonian counting statistics.

asymmetries in their shape, resulting in a time-evolving phase of the emitted photon pair [27]. Figure 1(b) shows a measurement of the entanglement fidelity in our experiment, using the procedure described in Appendix A and Refs. [25,28], with the characteristic oscillatory behavior. From the measured oscillation, we derive a splitting $S$ of $9.05 \pm 0.01 \mu \mathrm{eV}$. Owing to the improved QD density, we achieve a maximum fidelity of $92.0 \% \pm 0.2 \%$ to the static Bell $\Phi^{-}$state and $96.3 \% \pm 0.3 \%$ to the exact time-evolving state, which, to our knowledge, is the highest value ever reported from a QD emitter.

Figure 2 gives an overview of the main components of the quantum-relay setup. Alice uses a commercial tunable diode laser (linewidth, $400 \mathrm{kHz}$ ) with an attenuator and an electronic polarization controller (EPC) to send a stream of weak coherent input qubits in different polarization bases. Spectral overlap with the $2 X$ emission is controlled with a spectrometer and a fitting routine to overcome the limited instrument resolution, achieving an overall precision of $\pm 480 \mathrm{MHz}$.

At the relay station, the input mode and the $2 X$ photon from the QD emitter overlap in an unbalanced 0.18-dB coupler. Subsequent coincidence detection of photons at opposite outputs of a polarizing beam splitter (PBS) with detectors $D 1$ and $D 2$, corresponding to $H$ and $V$ polarization, projects the two interfering modes onto the Bell $\Psi^{+}$ state, heralding the teleportation of the input qubit into the 


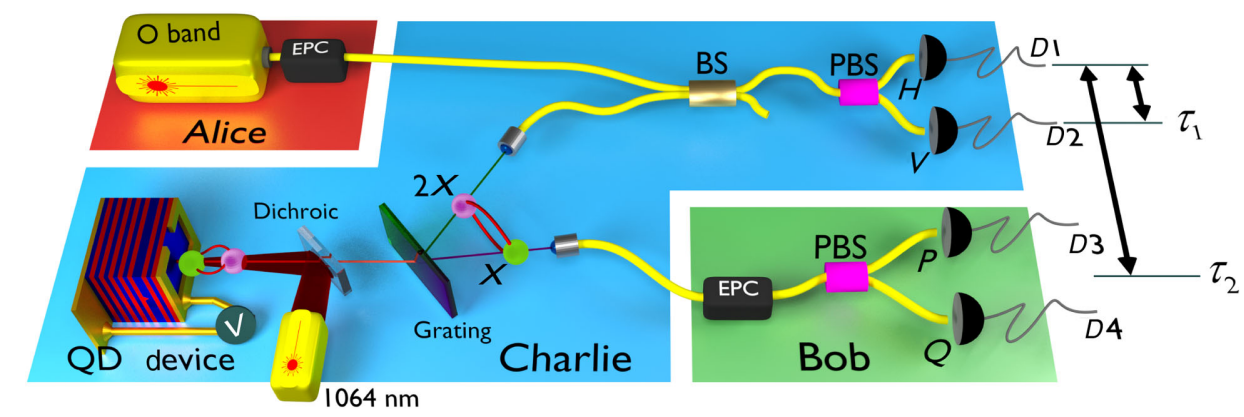

FIG. 2. Quantum relay setup including a QD entangled-photon-pair source. Alice encodes input states by the use of a telecommunication laser and an electronic polarization controller (EPC). Charlie performs a Bell-state measurement of the input and $2 X$ photons with an unbalanced beam splitter (BS), a polarizing beam splitter (PBS), and single-photon detectors $D 1$ and $D 2$. Bob uses an EPC, a PBS, and detectors $D 3$ and $D 4$ to analyze the output state of the $X$ photons in different polarization bases $(P$ and $Q)$.

polarization state of the $X$ photon (apart from a unitary transformation) [10].

The output is sent to the receiver (Bob), where it is analyzed in different polarization bases $(P$ and $Q)$, using an EPC, a PBS, and detectors $D 3$ and $D 4$. Photon detection is performed with superconducting nanowire single-photon detectors [29] and time-correlated single-photon-counting electronics with a measured cross-channel timing jitter of $70 \mathrm{ps}$. Count rates coming only from the photon-pair source during the experiments are typically $>200 \times 10^{3}$ counts $/ \mathrm{s}$ on each detector channel for $X$ and $>150 \times 10^{3}$ counts/s for $2 X$, the latter due to higher losses in the Bell-state measurement setup at Charlie.

For all measurements, the laser intensity is adjusted to achieve $90 \%$ of the detected $2 X$-photon rate. This optimal ratio is predicted from simulations based on the semiempirical model described in detail in Ref. [20] by taking all of the independently determined experimental parameters into account.

\section{RESULTS}

We start characterization of the quantum relay for implementation of a standard BB84 protocol [30] with Alice sending one of the four linear polarization states $H, V, D=$ $(H+V) / \sqrt{2}$, and $A=(H-V) / \sqrt{2}$. Coincidence detections between detectors $D 1$ and $D 2$ at Charlie herald the successful operation of the relay and have to be correlated with detections of the target photons at the receiver Bob, requiring the acquisition of three-photon coincidence maps as a function of time delays $\tau_{1}=t_{D 2}-t_{D 1}$ and $\tau_{2}=t_{\mathrm{Bob}}-t_{D 1}$. For each input state, we evaluate the fidelity of the measured output to the expected output as described in Appendix B.

Different physical mechanisms limit the maximum size of the temporal postselection window applicable for data evaluation. Coincidence detections along $\tau_{1}$ in the Bellstate measurement are sensitive to the interference visibility of the two distinct photon sources, which is influenced by the coherence time of $2 X$ photons and timing jitter. For discrimination along $\tau_{2}$, between the heralding event and detection of the target, the evolution of the phase of entangled photon pairs with the period $2 \pi \hbar / S=457 \pm$ 0.5 ps provides an upper limit.

Figure 3(a) shows the resulting mean teleportation fidelity for the protocol when varying the size of the postselection window along $\tau_{1}$ and $\tau_{2}$. The inset shows the point of highest significance, with an average teleportation fidelity of $87.9 \% \pm 1.1 \%$. This value exceeds the corresponding classical limit of $75 \%$ by more than 11 standard deviations and is above the $80 \%$ error-correction threshold required for a secure QKD [31]. When further reducing the time window at the cost of teleported qubits, we observe a maximum fidelity of $94.5 \% \pm 2.2 \%$ which, ideally, would contribute to 0.385 secure bits per detected threefold coincidence [32].

Practical implementations of a quantum relay should not only be compatible with passive telecommunication-network infrastructures but also with standard (nonscientific-grade) telecommunication laser sources. These components suffer from frequency drifts induced by temperature change and aging during continuous operation in nonlaboratory environments. We have investigated robustness of the relay against spectral detuning of the laser-generated input. Figure 3(b) displays the measured fidelity for input $D$ at different detunings from the resonance of QD emission. The results show that, for a frequency mismatch of $3 \mathrm{GHz}$, which is about the linewidth of the QD emission, we can still achieve fidelities of $80 \%$, enabling secure QKD applications, and, even for $6 \mathrm{GHz}$ of detuning, the fidelity exceeds the general classical limit of $2 / 3$. The results compare well with the displayed model simulation and indicate compatibility of the system with standard telecommunication light sources with typical spectral stabilities in the range of $1 \mathrm{GHz}$.

Finally, we characterize the general performance of the implemented photonic teleporter. For arbitrary pure input states,

$$
\left|\varphi_{\text {in }}\right\rangle=\cos \left(\frac{\theta}{2}\right)\left|H_{L}\right\rangle+e^{i \phi} \sin \left(\frac{\theta}{2}\right)\left|V_{L}\right\rangle,
$$

and, under the assumption of an ideal teleportation process, we expect the output for $\tau_{1}=0$ to be of the form 


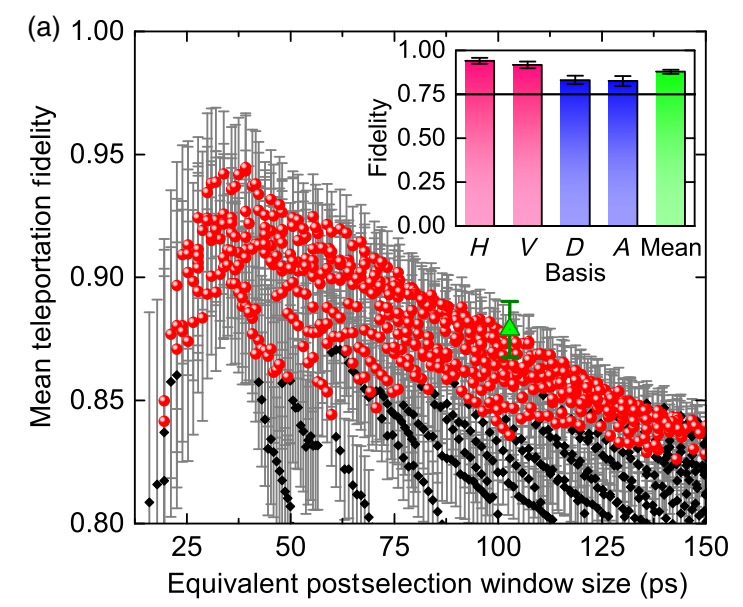

(b)

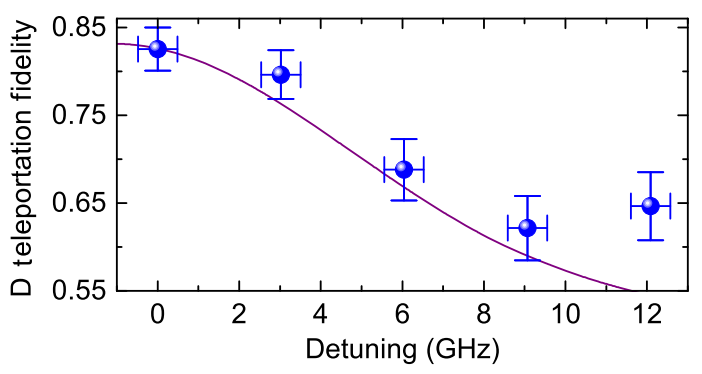

FIG. 3. Measured teleportation fidelities. (a) Average teleportation fidelity for implementation of a four-state protocol with Alice sending $H, V, D$, and $A$. Each point corresponds to a different size of the temporal postselection window $\Delta \tau_{1} \times \Delta \tau_{2}$ (in steps of $8 \mathrm{ps}$ ); data is plotted versus the square root of the window area. Red circles denote points with all individual teleportation fidelities above $75 \%$. The inset bar plot shows individual fidelities for $\Delta \tau_{1} \times \Delta \tau_{2}=88 \times 120 \mathrm{ps}$, indicated by the green triangle, with values of $94.1 \% \pm 1.7 \%(H), 91.7 \% \pm 1.9 \%(V)$, $83.1 \% \pm 2.5 \%(D), 82.5 \% \pm 2.8 \%(A)$, and $87.9 \% \pm 1.1 \%$ (mean). Errors are propagated from Poissonian statistics for the acquired raw data. (b) Teleportation fidelity with input $D$ for different detunings from the center wavelength of the relay interface ( $2 X$ photon). The solid line shows the corresponding simulation.

$\left|\varphi_{\text {out }}\left(\tau_{2}\right)\right\rangle=\cos \left(\frac{\theta}{2}\right)\left|V_{X}\right\rangle+e^{i\left(\phi-S \tau_{2} / \hbar\right)} \sin \left(\frac{\theta}{2}\right)\left|H_{X}\right\rangle$

Note that, in addition to a bit flip, the state is carrying the time-dependent phase acquired from the photon-pair source. Figure 4(a) shows the resulting oscillation for the input $D$ along $\tau_{1}=0$ in the three-photon coincidence space, measured when detecting the output at Bob in the diagonal basis ( $\sigma_{x}$ projection). The results compare well with the displayed model calculation [Fig. 4(b)] and enable a direct observation of the preservation of coherence in the teleportation process.

Rather than being a limitation, the time-evolving character of the output state provides an elegant way to perform quantum tomography. Full characterization of a polarization state generally requires three projective measurements
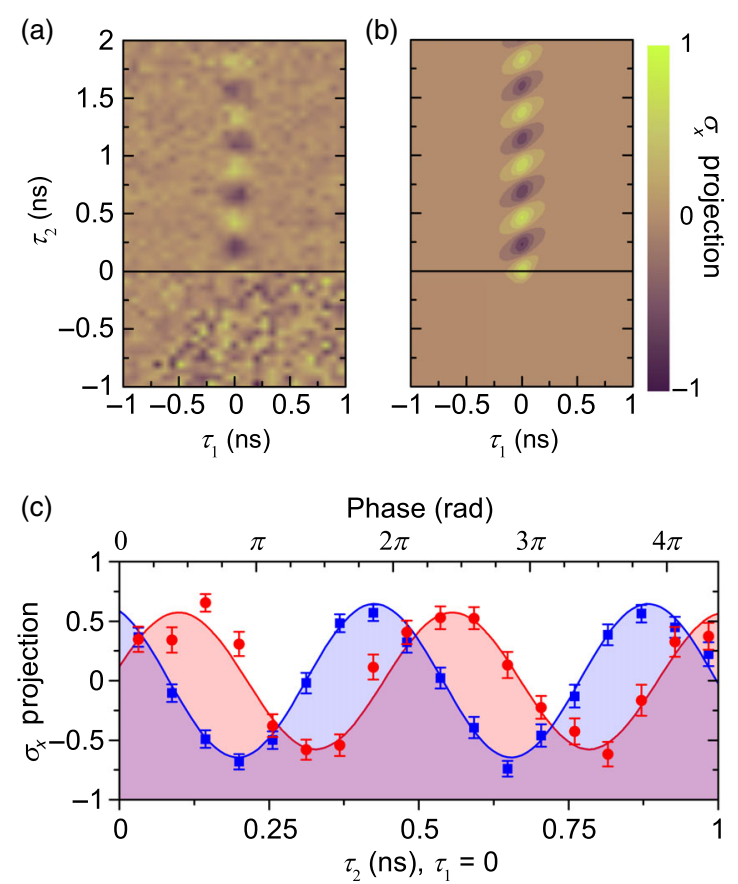

FIG. 4. Temporal evolution of teleported superposition states. (a) Measured $\sigma_{x}$ projection for the input $D$ with an oscillation along $\tau_{2} \geq 0$. (b) Corresponding simulation. (c) Cut through (a) along $\tau_{2}$ at $\tau_{1}=0$ for bin size $\Delta \tau_{1}=72 \mathrm{ps}$ and $\Delta \tau_{2}=56 \mathrm{ps}$. The blue squares are for the input $D$ (output $D$ ) and the red circles show the same measurement for the input $R$ (output $L$ ). Amplitude and phase are extracted with sinusoidal fits. There is a global fixed phase offset from $\tau_{2}=0$ due to decay from the $2 X$ state before reaching maximum population. Error bars are propagated from Poissonian statistics of the acquired raw data.

along the principal axes of the Poincaré sphere [33]. The temporal evolution in Eq. (3) corresponds to a rotation of the state in the equatorial plane of the Poincare sphere, enabling the projection onto that plane rather than a single axis by extracting amplitude and phase of the oscillation observable in the diagonal (DA) basis. Figure 4(c) compares the measured $D A$ projection (for details, see Appendix B) for inputs $R=(H+i V) / \sqrt{2}$ and $D$, showing the expected phase shift of $\pi / 2$ in the teleported state. Full tomography of the output at $\tau_{2}=0$ is completed by one additional projection onto the linear $(H V)$ basis in a separate measurement and by using the methods described in Ref. [33]. For the analysis, for each measurement basis, a total of $1.008 \mathrm{~ns}$ of data along $\tau_{2}$ is taken into account, corresponding to more than two full rotations of the state.

The characterization of a quantum process can be described by the process matrix $\chi$. For single qubits, the mapping process $\epsilon(\rho)$ for an input state represented by the density matrix $\rho$ is conveniently expressed as $\epsilon(\rho)=$ $\sum_{m n} \sigma_{m} \rho \sigma_{n} \chi_{m n}$, with $\sigma_{i}=\left\{\mathbb{1}, \sigma_{x}, \sigma_{y}, \sigma_{z}\right\}$ being the identity and Pauli operators. Using the methods described before, we did state tomography of the output for inputs $H, V, D$, and $R$ enabling quantum-process tomography following 
(a)

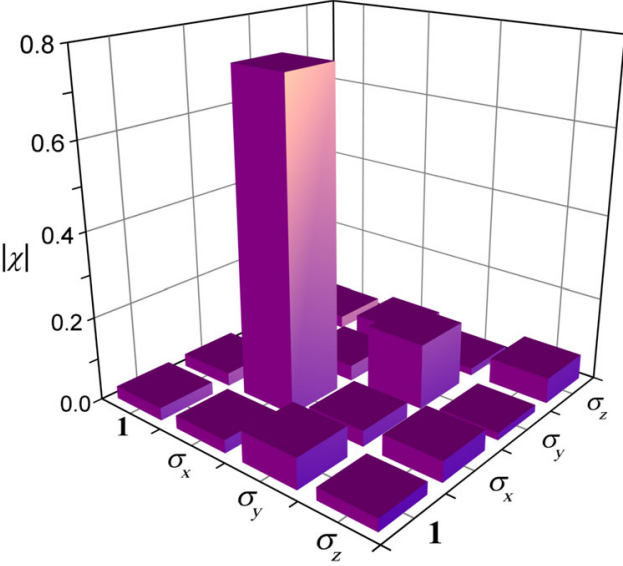

(b)

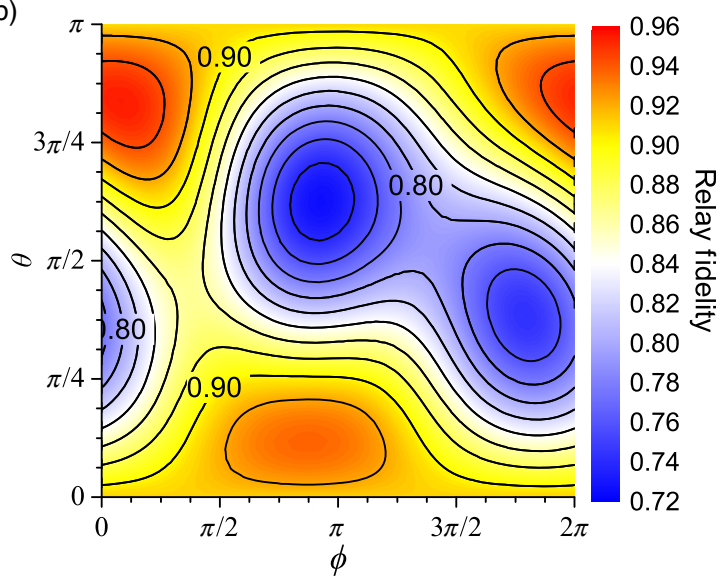

FIG. 5. Quantum-process tomography. (a) Modulus of the reconstructed process matrix with a $\sigma_{x}$ component of $0.754 \pm 0.016$. (b) Calculation of relay fidelity based on the process matrix for arbitrary pure input states parametrized by angles $\theta$ and $\phi$.

the procedure described in Ref. [34]. Figure 5(a) shows the reconstructed process matrix with the dominant contribution from the bit-flip operator $\sigma_{x}$. We deduce a process fidelity of $75.4 \% \pm 1.6 \%$, corresponding to an average teleportation gate fidelity of $83.6 \% \pm 1.1 \%$ [35] for arbitrary input states. It has to be emphasized that this value is to be considered as a lower bound due to the large time window along $\tau_{2}$ (more than $1 \mathrm{~ns}$ ) taken into account for the tomography.

Based on the reconstructed process matrix, we calculate the relay fidelity for arbitrary pure states as described by Eq. (2), providing a better insight into the photonic teleportation process. Figure 5(b) shows the fidelity of the experimentally expected output to the corresponding ideal output state, under the assumption that the ideal teleporter is described by a pure $\sigma_{x}$ operation.

We observe high fidelities exceeding $90 \%$ close to the polar states $H$ and $V(\theta=0, \pi)$. As these states correspond to the measurement basis of the Bell setup at Charlie, the gate is mainly limited by the entanglement fidelity of the photonpair source, the extinction ratio of the polarization detection optics, and dark counts. The situation is rather different when approaching superposition states with $\theta=\pi / 2$. Here, two-photon interference comes into play which is dominated by the Poissonian statistics of the input qubits $[20,26,36]$ and not the relay itself, lowering the teleportation fidelities. Still, minimum values are safely above the classical limit of $2 / 3$, indicating possible quantum teleportation for arbitrary input states. Furthermore, at no point does the fidelity drop below $72.4 \%$, the threshold required for secure implementation of six-state protocols $[31,37]$.

\section{CONCLUSION}

In this paper, we demonstrate quantum teleportation of weak coherent telecommunication-wavelength polarization qubits making use of a semiconductor quantum-dot entangled-photon-pair source. We characterize the performance for implementation of a four-state protocol in a quantum relay, with fidelities compatible with required security thresholds and maximum values exceeding $90 \%$. We further show robustness of the teleportation against frequency drifts of laser-generated input states, as they are expected for off-the-shelf coherent telecommunication light sources. The time-evolving character of the entangled photon-pair source enables us to directly observe the phase coherence of the photonic teleportation process. Finally, we take advantage of this effect for quantum-state tomography of teleported states and reconstruct the process matrix of the relay, evidencing quantum teleportation for arbitrary pure input states.

The exclusive operation of the demonstrated system at telecommunication wavelength combined with subPoissonian statistics of the entangled photon-pair source represents a promising platform. Apart from the challenges involved in sending entangled qubits over a long optical fiber, generally requiring the implementation of sophisticated feedback systems, this approach is, in principle, cascadable as required for long-haul communication in a quantum-relay scheme [7]. Not only is it compatible with existing communication infrastructures, it also provides intrinsic security without the need for additional security protocols, which might prove advantageous for scalable implementation. Furthermore, the prospect of teleporting arbitrary input states shows great potential, beyond cryptography applications, for the broad spectrum of emerging photonic quantum-information technologies. The results provide solid evidence that semiconductor telecommunication light sources can be considered a serious alternative to existing well-established approaches for the generation of photon entanglement at telecommunication wavelength, an important step for the development of a standard quantum-relay technology in the future.

\section{ACKNOWLEDGMENTS}

The authors acknowledge partial financial support from the Engineering and Physical Sciences Research Council 
and the EPSRC Quantum Technology Hub in Quantum Communications. M. F. acknowledges support from the EPSRC CDT in Photonic Systems Development (EP/ G037256/1). J. H. would like to thank M. Lucamarini for the theoretical support and T. Müller for the technical assistance.

\section{APPENDIX A: CHARACTERIZATION OF ENTANGLEMENT}

The fidelity of entangled photon pairs emitted from the QD to a maximally entangled Bell-like state of the form

$$
|\Phi(\phi)\rangle=\frac{1}{\sqrt{2}}[|H H\rangle+\exp (i \phi)|V V\rangle]
$$

can be calculated as $[25,28]$

$$
\begin{aligned}
F(\phi)= & \frac{1}{4}\left[1+C_{H V}+\left(C_{D A}-C_{L R}\right) \cos \phi\right. \\
& \left.+\left(C_{E_{L D} E_{R A}}-C_{E_{L A} E_{R D}}\right) \sin \phi\right],
\end{aligned}
$$

where $\phi=0, \pi$ corresponds to the Bell $\Phi^{+}$and $\Phi^{-}$state and $\phi=S \tau / \hbar$ represents the expected time-evolving state (A1) characterized by the splitting parameter $S$. The indices $H, V$, $D, A, R$, and $L$ denote the principal polarizations spanning the Poincare sphere. The indices $E_{L D}, E_{L A}, E_{R A}$, and $E_{R D}$ denote the corresponding elliptical states lying in the equatorial plane. Please note that, since we are interested in the fidelity to the expected state (A1), reconstruction of the full density matrix of the two-photon state, generally requiring 16 independent measurements, is not necessary. Instead, Eq. (A2) implies only ten measurements for the exact evaluation of the fidelity of interest $[25,28]$.

The coefficients $C_{P Q}$ for polarization correlation measurements of $2 X$ and $X$ photons in polarization bases $P Q$ are calculated as

$$
C_{P Q}^{e n t}\left(\tau_{2}\right)=\frac{g_{P_{2 X} P_{X}}^{(2)}\left(\tau_{2}\right)-g_{P_{2 X} Q_{X}}^{(2)}\left(\tau_{2}\right)}{g_{P_{2 X} P_{X}}^{(2)}\left(\tau_{2}\right)+g_{P_{2 X} Q_{X}}^{(2)}\left(\tau_{2}\right)}
$$

after measurement of two-photon correlations for the coand cross-polarized cases $\left(g_{P_{2 X} P_{X}}^{(2)}\right.$ and $g_{P_{2 X}}^{(2)} Q_{X}$, respectively).

For the data presented in Fig. 1(b), we measure secondorder photon correlations setting the polarization discrimination setup at Charlie and Bob to the bases $H V, D A, R L$, $E_{L D} E_{R A}$, and $E_{L A} E_{R D}$. The laser input from Alice is turned off during these measurements. The spin splitting has been determined in a separate measurement independently. No background subtraction is applied when analyzing the data.

\section{APPENDIX B: CHARACTERIZATION OF TELEPORTATION}

For successful coincidence detection between detectors $D 1$ and $D 2$ in the Bell-state measurement, the implemented teleportation circuit transforms arbitrary input states as described by Eqs. (2) and (3). Neglecting the time dependence of the output by evaluating the state at the time $\tau=0$, this results in the following transformations between input and output: $H \rightarrow V, V \rightarrow H, D \rightarrow D, A \rightarrow A, R \rightarrow L$, and $L \rightarrow R$.

Teleportation is verified by measuring third-order correlation functions $g_{H V P_{X}}^{(3)}\left(\tau_{1}, \tau_{2}\right)$ of the photons detected at Charlie's detectors $D 1$ and $D 2$, corresponding to $H$ and $V$, and either one of Bob's detectors D3 and D4, corresponding to polarization $P_{X}$ or $Q_{X}$ (with $Q_{X}$ being orthogonal to $P_{X}$ ). Raw data are recorded with 1-ps timing resolution and rebinned on an 8-ps grid before later analysis. The teleportation fidelity, defined as the overlap with the desired output $P$, is then calculated as

$$
F_{P}\left(\tau_{1}, \tau_{2}\right)=\frac{g_{H V P_{X}}^{(3)}\left(\tau_{1}, \tau_{2}\right)}{g_{H V P_{X}}^{(3)}\left(\tau_{1}, \tau_{2}\right)+g_{H V Q_{X}}^{(3)}\left(\tau_{1}, \tau_{2}\right)} .
$$

For the state tomography measurements, data are acquired similarly, with Bob detecting in the $H V$ and $D A$ bases. The projection of the output state along the axis $P Q$ on the Poincaré sphere is then calculated as

$$
C_{P Q}^{t e l}\left(\tau_{1}, \tau_{2}\right)=\frac{g_{H V P_{X}}^{(3)}\left(\tau_{1}, \tau_{2}\right)-g_{H V Q_{X}}^{(3)}\left(\tau_{1}, \tau_{2}\right)}{g_{H V P_{X}}^{(3)}\left(\tau_{1}, \tau_{2}\right)+g_{H V Q_{X}}^{(3)}\left(\tau_{1}, \tau_{2}\right)} .
$$

[1] Artur K. Ekert, Quantum Cryptography Based on Bell's Theorem, Phys. Rev. Lett. 67, 661 (1991).

[2] Nicolas Gisin, Grégoire Ribordy, Wolfgang Tittel, and Hugo Zbinden, Quantum cryptography, Rev. Mod. Phys. 74, 145 (2002).

[3] H. J. Kimble, The quantum internet, Nature (London) 453, 1023 (2008).

[4] Hoi-Kwong Lo, Xiongfeng Ma, and Kai Chen, Decoy State Quantum Key Distribution, Phys. Rev. Lett. 94, 230504 (2005).

[5] Danna Rosenberg, Jim W. Harrington, Patrick R. Rice, Philip A. Hiskett, Charles G. Peterson, Richard J. Hughes, Adriana E. Lita, Sae Woo Nam, and Jane E. Nordholt, LongDistance Decoy-State Quantum Key Distribution in Optical Fiber, Phys. Rev. Lett. 98, 010503 (2007).

[6] Shuang Wang, Wei Chen, Jun-Fu Guo, Zhen-Qiang Yin, Hong-Wei Li, Zheng Zhou, Guang-Can Guo, and Zheng-Fu Han, $2 \mathrm{GHz}$ clock quantum key distribution over $260 \mathrm{~km}$ of standard telecom fiber, Opt. Lett. 37, 1008 (2012).

[7] B. C. Jacobs, T. B. Pittman, and J. D. Franson, Quantum relays and noise suppression using linear optics, Phys. Rev. A 66, 052307 (2002).

[8] Koji Azuma, Kiyoshi Tamaki, and Hoi-Kwong Lo, Allphotonic quantum repeaters, Nat. Commun. 6, 6787 (2015).

[9] H.-J. Briegel, W. Dür, J. I. Cirac, and P. Zoller, Quantum Repeaters: The Role of Imperfect Local Operations in Quantum Communication, Phys. Rev. Lett. 81, 5932 (1998). 
[10] C. H. Bennett, G. Brassard, C. Crépeau, R. Jozsa, A. Peres, and W. K. Wootters, Teleporting an Unknown Quantum State via Dual Classical and Einstein-Podolsky-Rosen Channels, Phys. Rev. Lett. 70, 1895 (1993).

[11] Hoi-Kwong Lo and H. F. Chau, Unconditional security of quantum key distribution over arbitrarily long distances, Science 283, 2050 (1999).

[12] D. Bouwmeester, J.-W. Pan, K. Mattle, M. Eibl, H. Weinfurter, and A. Zeilinger, Experimental quantum teleportation, Nature (London) 390, 575 (1997).

[13] Q.-C. Sun, Y.-L. Mao, S.-J. Chen, W. Zhang, Y.-F. Jiang, Y.-B. Zhang, W.-J. Zhang, S. Miki, T. Yamashita, H. Terai, X. Jiang, T.-Y. Chen, L.-X. You, X.-F. Chen, Z. Wang, J.-Y. Fan, Q. Zhang, and J.-W. Pan, Quantum teleportation with independent sources and prior entanglement distribution over a network, Nat. Photonics 10, 671 (2016).

[14] R. Valivarthi, M. Grimau Puigibert, Q. Zhou, G. H. Aguilar, V. B. Verma, F. Marsili, M. D. Shaw, S. W. Nam, D. Oblak, and W. Tittel, Quantum teleportation across a metropolitan fibre network, Nat. Photonics 10, 676 (2016).

[15] Oliver Benson, Charles Santori, Matthew Pelton, and Yoshihisa Yamamoto, Regulated and Entangled Photons from a Single Quantum Dot, Phys. Rev. Lett. 84, 2513 (2000).

[16] P. Michler, A. Kiraz, C. Becher, W. V. Schoenfeld, P. M. Petroff, Lidong Zhang, E. Hu, and A. Imamoglu, A quantum dot single-photon turnstile device, Science 290, 2282 (2000).

[17] M. Müller, S. Bounouar, K. D. Jöns, M. Glässl, and P. Michler, On-demand generation of indistinguishable polarization-entangled photon pairs, Nat. Photonics 8, 224 (2014).

[18] R. M. Stevenson, R. J. Young, P. Atkinson, K. Cooper, D. A. Ritchie, and A. J. Shields, A semiconductor source of triggered entangled photon pairs, Nature (London) 439, 179 (2006).

[19] C. L. Salter, R. M. Stevenson, I. Farrer, C. A. Nicoll, D. A. Ritchie, and A.J. Shields, An entangled-light-emitting diode, Nature (London) 465, 594 (2010).

[20] C. Varnava, R. M. Stevenson, J. Nilsson, J. Skiba-Szymanska, B. Dzurňák, M. Lucamarini, R. V. Penty, I. Farrer, D. A. Ritchie, and A. J. Shields, An entangled-LED-driven quantum relay over $1 \mathrm{~km}$, npj Quantum Inf. 2, 16006 (2016).

[21] B. Alloing, C. Zinoni, V. Zwiller, L. H. Li, C. Monat, M. Gobet, G. Buchs, A. Fiore, E. Pelucchi, and E. Kapon, Growth and characterization of single quantum dots emitting at $1300 \mathrm{~nm}$, Appl. Phys. Lett. 86, 101908 (2005).

[22] M. Benyoucef, M. Yacob, J. P. Reithmaier, J. Kettler, and P. Michler, Telecom-wavelength $(1.5 \mu \mathrm{m})$ single-photon emission from InP-based quantum dots, Appl. Phys. Lett. 103, 162101 (2013).

[23] M. B. Ward, O. Z. Karimov, D. C. Unitt, Z. L. Yuan, P. See, D. G. Gevaux, A. J. Shields, P. Atkinson, and D. A. Ritchie,
On-demand single-photon source for $1.3 \mathrm{~m}$ telecom fiber, Appl. Phys. Lett. 86, 201111 (2005).

[24] J. Skiba-Szymanska, R. M. Stevenson, C. Varnava, M. Felle, J. Huwer, T. Müller, A. J. Bennett, J. P. Lee, I. Farrer, A. B. Krysa, P. Spencer, L. E. Goff, D. A. Ritchie, J. Heffernan, and A. J. Shields, Universal Growth Scheme for Quantum Dots with Low Fine-Structure Splitting at Various Emission Wavelengths, Phys. Rev. Applied 8, 014013 (2017).

[25] M. B. Ward, M. C. Dean, R. M. Stevenson, A. J. Bennett, D. J. P. Ellis, K. Cooper, I. Farrer, C. A. Nicoll, D. A. Ritchie, and A. J. Shields, Coherent dynamics of a telecomwavelength entangled photon source, Nat. Commun. 5, 3316 (2014).

[26] M. Felle, J. Huwer, R. M. Stevenson, J. Skiba-Szymanska, M. B. Ward, I. Farrer, R. V. Penty, D. A. Ritchie, and A. J. Shields, Interference with a quantum dot single-photon source and a laser at telecom wavelength, Appl. Phys. Lett. 107, 131106 (2015).

[27] R. M. Stevenson, A. J. Hudson, A. J. Bennett, R. J. Young, C. A. Nicoll, D. A. Ritchie, and A. J. Shields, Evolution of Entanglement between Distinguishable Light States, Phys. Rev. Lett. 101, 170501 (2008).

[28] P. Michler, Single Semiconductor Quantum Dots (Springer, New York, 2009).

[29] Single Quantum.

[30] C. H. Bennett and G. Brassard, in Proceedings of the IEEE International Conference on Computers, Systems, and Signal Processing, Bangalore, India, 1984 (IEEE, New York, 1984), p. 175.

[31] H. F. Chau, Practical scheme to share a secret key through a quantum channel with a $27.6 \%$ bit error rate, Phys. Rev. A 66, 060302 (2002).

[32] Peter W. Shor and John Preskill, Simple Proof of Security of the BB84 Quantum Key Distribution Protocol, Phys. Rev. Lett. 85, 441 (2000).

[33] D. F. V. James, P. G. Kwiat, W. J. Munro, and A. G. White, Measurement of qubits, Phys. Rev. A 64, 052312 (2001).

[34] M. A. Nielsen and I. L. Chuang, Quantum Computation and Quantum Information (Cambridge University Press, Cambridge, England, 2000).

[35] M. A. Nielsen, A simple formula for the average gate fidelity of a quantum dynamical operation, Phys. Lett. A 303, 249 (2002).

[36] R. M. Stevenson, J. Nilsson, A. J. Bennett, J. SkibaSzymanska, I. Farrer, D. A. Ritchie, and A. J. Shields, Quantum teleportation of laser-generated photons with an entangled-light-emitting diode, Nat. Commun. 4, 2859 (2013).

[37] Dagmar Bruß, Optimal Eavesdropping in Quantum Cryptography with Six States, Phys. Rev. Lett. 81, 3018 (1998). 